\title{
A Survey on Patient Queue Management System
}

\author{
Prof. Neha Titarmare, Prof. Ashwini Yerlekar
}

\author{
Computer Science \& Engineering Department, Rajiv Gandhi College Of Engineering \& Research, Wanadongri, Hingna \\ Road, Nagpur, India
}

\begin{abstract}
In this paper, we study the various types of Queue and Queue Management System. Queue system can successfully reduce waiting time of the patients in the hospitals. We aim to implement a model that initializes alert notification via SMS to patients of a hospital. It will minimize the queue of patients in the waiting area of hospital and also patient can book an appointment from anywhere at a given time. Patients can book an appointment via android application. and accordingly patients and also it will provide navigation towards that nearest hospital. Hence, for developing such a model a survey has been done on various types of queues.
\end{abstract}

Keywords - Queue, Queue Management System.

\section{INTRODUCTION}

Time is a quantity that should be efficiently managed. Standing in a long queue consumes a lot of time of patients and so, the Patient Queue Management System provides the solution for it. This Patient Queue Management System will reduce the burden of waiting in a long queue. Most hospitals and clinics try to improve their wait time by scheduling appointments. Appointments seem like a logical, clear-cut solution you register in advance and come at the agreed time. It does so through relevant information and personalization. Hence we have come up with an idea of developing a GSM device and android application which will reduce the waiting time by providing alert notifications to the patients.

The patient will be able to choose the specific doctor for a specific treatment. Also the patients, who are unaware about the hospitals location, can easily use the android application which will provide the navigation to that hospital.

The patient would get simply registered by just giving a miss call and accordingly patient will get the alert notifications and details about their appointment which would include the token number.

\section{LITERATURE SURVEY}

\section{a) Introduction}

A queue management system is the organization of queues of people within a retail or public sector department. It can be either reactive through a system that can organize the existing queue or proactive through queue management statistics gathering system, so that the trends can be identified and anticipated. People that join queue in a standing line queue are directed to the next position by the system or be issued with a ticket. With a ticketed system, customers are took out of the standing line queue, which can give comfort and less stress for the customers as well as their turns are not neglected. This queuing environment is an essential part of our daily lives and it is important for manufacturer to build the most cost-effective queuing solution.

\section{b) Types of Queue}

There are two types of queue, which are structured and unstructured queues. Structured queue is in a fixed form and people that are included are in predictable position. We can see this at a supermarket paying counter and some other retail locations such as banks and post offices. This type of queue systems is often being set up to manage ticket ranking for a service with identification and thus enable a stress-free waiting. Extending the various possibilities, some of these systems are planned reception by appointment or remotely rank allocation on Smartphone or through SMS. On the other hand, in unstructured queue people form a queue in unpredictable manner and at different locations and directions. Such queues are visible in certain cases like in some forms of retail, taxi queues, ATMs and at period of high demand in many situations. The prevailing solutions for this issue are Rank allocation for services, pages or RFID badges or reading the customer card. In fact, it is hard to implement a way of structuring these queues to be successful. The reason is that one cannot easily calculate the behavior of man.

\section{c) Queue Management Techniques}

Various queue management techniques exist like physical barrier, signage and signaling systems and automatic queue measurement system. Physical barrier is aim at guiding queue formation and organizing it in a neat way. While signage and signaling systems aims to provide information to people, queuing and aid efficient queue formation flow as well as setting service expectations. Differently, automatic queue measurement system uses a variety of measurement technologies, which predict and measure lengths and waiting times, which provide management information to help service levels and resource deployment. 


\section{d) Queue Management Concept}

A lot of research has been done previously on the queue management concepts such as Shortest Processed First (SPF), First Come First Serve (FCFS), Single Queue (SQ), Multiple Queue (MQ), Diffuse Queue (DQ) and Head of Queue (HQ).

SPF works as a scheduling policy that requires lesser time execution to process first. In a supermarket, a specific paying counter only processes transaction where a customer is buying less than 10 goods. SPF works well due to its simplicity and it reduces the average amount of waiting time for each process. However, the setback is that it requires a long time to complete if short process continually adds and customers do observe unfairness from the system. It is crucial to demonstrate why customers are serving in that order and ensure the understanding of customers to see the logic of this alternative approach. On the other hand, FCFS is a method that deals the oldest entry being processed first. FCFS behavior is where people leave the queue in the order, in which they arrive. It is the fairest service provision where all customers think of themselves as equal.

The single queue (SQ) is the familiar zigzag format. Each person waiting is served in turn and the format discourages pushing in. The queue also provides assurance to customers that they will be served in a fair manner while the queue is progressing. Besides that, the multiple queues (MQ) is the improvement of SQ, it is essential to use this method when handling larger amount of people. This queue is the format that is always being used at the supermarket. Other than that is the diffuse queue, DQ, which has no formal queue line but customers registered place is in the process with a ticket. Last, is the head queue (HQ) which proposes that the next person to be served waits in a single queue environment. This method is important when the number of checkout is higher than five. It is vital that customers are able to see along the line of service position to avoid significant gaps in service provision. In order to maintain fairness in this system, several rules have been set:

- Queue must be fair and be managed systematically that cannot be allowed to descend into a chaotic situation.

- Perception of waiting time should be managed.

- The process must be clearly identified; start and ends must be visible.

- The process must include positive feedback of progress.

\section{e) Advantages of Queue Management System}

Queue management system gives benefits either to customer service provider or to the customer itself. The benefits can be directly or indirectly to the system. They are as follows :

- Keeps track and forecast the flow of customers.

- Optimum utilization of staff forecast.

- Constant monitoring the staff's performance.
- Enhance productivity and morale of the staff, as operations are efficient and systematic.

- Gives flexibility in dealing with customers.

- Increase service reliability, as customers are treated fairly and efficient.

- Producing statistical reports, which facilitate top Management's decision making process.

\section{f) Existing Queue Management System}

Many products are available in the market produced by various queue management companies for delivering optimum customer service. Some of these products are Stand Alone Queue System and Centralized Control Queue System.

\section{I]. Stand Alone Queue System}

Stand Alone Queue System (SAQS) design is based on First Come First Serve, FCFS queue model, where there is only one service counter operation. All customers will be managed at the single counter. This system operates by calling or displaying number in sequential or randomized order and the customers will be treated fairly. The SAQS is performing well in a single department, service operation environment such as clinic.

\section{II]. Advance Queue System}

Advance Queue System (AQS) based on SAQS design where additional service counters are added to give flexibility in queue system process. This system can support up to 32 service counters and additional of 60 counters. It also provides useful queue features as well as comprehensive reports. Besides that, it allows manager of real-time monitoring status for the queue management analysis. AQS is performing well in bank, hospital or any organization that has multiple department service operation.

\section{III]. Centralized Control Queue System}

Centralized Control Queue System (CCQS) design is used for higher range of customers in different department. This system has the capability to support up to 20 departments where each department can have up to 32 service counters and 60 counters. CCQS is network compatible because each department has been located at different part of a building or even in geographical area. Thus, CCQS is connected through LAN or Internet and it also provides real-time status monitoring.

\section{g) Customer Flow Management}

There are few managers for service providers who know what happens with their customers throughout the whole interaction process in real-time. The Head of Operations often does not know the flow of customers well, if the supply and production managers know the flow of material and 
product in detail. The flow of customers is more important than the flow of material and products. Also, the flow of customers is more difficult to monitor and control. For any service provider, properly knowing the customers is an absolute key success factor. Therefore, Customer Flow Management (CFM) is managing the flow of customers and their experience from initial contact to final service delivery. The Customer Flow Management process consist of several phases including pre-arrival, arrival, queuing or waiting, serving, post-serving and managing. It is view of the entirety of the customer service operation, the resulting framework for making informed business decisions and the frames the boundaries of CFM.

\section{I]. Pre-arrival}

CFM can start before the customers physically visits the shop or service center by implementing a method to book appointments before arrival. These reduces the time spent waiting by the customer and produce a positive impact on the customer's service experience.

\section{II]. Arrival}

Customers need to be in place in an appropriate queue on arrival. Customer Flow Management stresses the possibility of segmenting the customers in different queues rather than entering all customers in the same queue.

\section{III]. Queuing/waiting}

Most customers will endure a period of waiting after queue entry. A balanced and controlled waiting period is the desired optimum results of any managers.

No one wants to have a completely empty waiting area as it reflected that you are overstaffed or impression of abandonment. Equally, too many customers waiting are simply as off-putting. In the case of a hospital or public service center, certain citizens might not accept it as they can demonstrate this during elections. CFM can help managers get the balance rightly by improving staff planning and by adding more flexibility to the process.

\section{[IV]. Serving}

When calling the customer forward, staff can start preparations if the service chooses to identify and tracking customer's history before the customer actually arrives at the service point.

\section{V]. Post-serving}

After a customer has been served, a case handling function can continue to manage the case throughout its lifetime if needed and each step is document and process.

VI]. Managing
Managers can use the gathered data in CFM process to evaluate the current processes. Reports can be generated on employee-customer interactions, service times and customer wait times. Operational inefficiencies can be identified and addressed through process changes or training.

\section{ADVANTAGES OF THE SYSTEM}

The system offers several attractive features like:

1. Convenience-SMS technology is easy to use and learn and can be accessed easily when needed.

2. Accessibility - instructions can be sent to the microcontroller to be controlled and monitored from any location provided there is the existence of an active GSM network or control from anywhere in the world if cellular coverage is available.

3. Portability - a microcontroller can be controlled and monitored from any GSM phone that supports SMS. Considering the fact that most GSM phones support SMS, the system is therefore highly portable.

4. Saves Time - an SMS based remote monitoring and computer control system saves time as the user is not required to gain access to an internet connection or make a dedicated connection to the computer to be controlled as opposed to a Bluetooth-based system or an Internet based system.

5. Cheaper - SMS services are generally cheap and are sometimes provided for free (at least for certain periods) by service providers. Furthermore, most service providers do not charge users for receiving SMS.

6. Mobility - User and/or system administrators are more likely to have their phones with them at all times than they are likely to physically be in front of their computers. An SMS based system, therefore, enables them to have ubiquitous access to the computer to be controlled and monitored.

7. Acknowledgement- The System issues acknowledgement about execution of command to user.

8. To use SMS service and give commands from user for control.

\section{CONCLUSION}

Patient queue management system starts with the understanding of the queue system itself, which is very crucial to broadening the horizon of understanding. Then, the consideration of the control strategy and component to be used plays important role as guidelines to accomplish this project. In addition, several articles have been a review to investigate the current approaches for queue management system. Although the current approaches have proven to ease and give benefits to service providers, yet there is space for improvement in order for a queue system function efficiently. 
REFERENCES

[1] Ahmed, S. A. \& Huda, K.T. (2011), Automatic Queuing Model for Banking Applications , International Journal of Advanced Computer Science and Applications (IJACSA), 2(7).

[2] Allen, A. (1990), Probability, Statistics and Queuing Theory with Compute Science Applications , Academic Press Inc., Second Edition, 1990.

[3] Goluby, B. \& Preston McAfeez, R. (2011), Firms, Queues and Coffee Breaks: A Flow Model of Corporate Activity with Delays, Springer-Verlag 15, 59-89.

[4] Gurumurthi, S. \& Benjaafar, S. (2004), Modelling and Analysis of Flexibile Queuing Systems, Wiley Periodicals, Inc. DOI 10.1002/nav.20020.

[5] Hossain, Md. B., Hossain, Md. N., Hossen, Md. M. \& Rahman, Md. H. (2011), Design and Development of Microcontroller Based Electronic Queue Control Systems, Proceeding of the 2011 IEEE Students' Technology Symposium.

[6] Koole, G. \& Mandelbaum, A. (2002), Queuing Models of Call Centers, An Introduction, Ann Oper Res 113, 41-59.

[7] Lustsik, O. (2003), E- Banking in Estonia: Reasons and Benefits of the Rapid Growth, University of Tartu, Kroon and Economy 3, 24-36

[8] Maister, David H. (2005), the psychology of Waiting Lines.

[9] Mobarek, A. (2007), E- Banking Practices and Customer Satisfaction- A Case study in Botswana, 20th

[10] Australasian Finance \& Banking Conference.

[11] Mohamad Fazli Alias (2007), Front Desk Customer Service for Queue Management System.

[12] Q-Matic AB (2008), an Introduction to Customer Flow Management, Version L1.5.

[13] Sanjay, Bose K. (2002), an Introduction to Queuing Systems, Springer.

[14] Tocci, R. J. (2000), Digital Systems. New Jersey: Prentice -Hall, 7th edition.

[15] Willig, a. (1999), A Short Introduction to Queuing Theory, Technical University Berlin, Telecommunication Networks Group Sekr. FT 5-2, Berlin.

[16]Zhang, No \& Tay (2000). Discrete-event simulation

[17] of queuing systems, published in the Proceedings of the sixth youth conference, Ministry of Education, Singapore. 